\title{
La gestión de operaciones como herramienta generadora de ventajas competitivas en la industria alimentaria vegetariana
}

\section{Operation Management as Generator a Competitive Advantages in the Vegetarian Food Industry.}

Rodrigo Andrés Escandón Cueva. ${ }^{1}$, Juan Carlos Erazo Álvarez. ${ }^{2}$, Cecilia Ivonne Narváez Zurita. ${ }^{3}$, \& Jorge Edwin Ormaza Andrade. ${ }^{4}$

\section{DOI: https://doi.org/10.33262/visionariodigital.v3i2.2.597}

\begin{abstract}
.
In recent years, there has been a worldwide trend, that seeks to improve health and lifestyle, especially in younger generations. One of the most common ways to do so is by changing feeding habits. Reducing or leaving meat-based food is not only considered a healthier way of alimentation but an environmentally-friendly practice; consequently, most people have become vegetarians in recent years. This study was developed through a non-experimental design using a qualitative-quantitative approach, and a sequential, descriptive - explanatory research (DEXPLIS). The data analysis methods used for the theoretical framework were inductive, deductive, and analytic-synthetic. The main problem found in the company, Grantor located in Deled, Ecuador, after analyzing surveys and interviews to the main distributors and production manager was an improper estimate over the production costs, inventory accumulation,
\end{abstract}

\footnotetext{
${ }^{1}$ Universidad Católica de Cuenca, Posgradista Maestría de Administración de Empresas, Cuenca, Ecuador, raescandonc@psg.ucacue.edu.ec

2 Universidad Católica de Cuenca, Sub Dirección de Posgrados, Cuenca, Ecuador. jcerazo@ucacue.edu.ec

3 Universidad Católica de Cuenca, Sub Dirección de Posgrados, Cuenca, Ecuador. inarvaez@ucacue.edu.ec

4 Universidad Católica de Cuenca, Sub Dirección de Posgrados, Cuenca, Ecuador. jormaza@ucacue.edu.ec
} 
inefficient production policies, and the high cost of paying overtime. From these conclusions comes the need to design a management approach based on a linear programming model that guarantees the optimum cost-benefit result.

Keywords: production, operation management, linear programing, natural products, production costs.

\section{Resumen.}

Existe en la actualidad una marcada tendencia a nivel mundial para mejorar la salud y el estilo de vida de las personas, sobre todo en nuevas generaciones. Una de las principales maneras de conseguir esto es cambiando los hábitos alimenticios tradicionales, y es por ello que el vegetarianismo ha ganado una particular importancia en años recientes, ya que reducir o eliminar el consumo de productos de origen animal no solo implica una alimentación más saludable sino también un hábito más amigable con el medio ambiente. La investigación se desarrolló bajo un diseño no experimental, con enfoque cuali-cuantitativo y un alcance descriptivo - explicativo secuencial (DEXPLIS). Los métodos utilizados para el análisis de la literatura fueron el inductivodeductivo y el analítico-sintético. Entre los principales resultados obtenidos de la aplicación de encuestas a los distribuidores de la empresa GranOrt ubicada en el cantón Deleg, Ecuador, y de la entrevista al jefe de producción de la empresa, se determinó el deficiente cálculo de los costos de producción, altos volúmenes de inventario, inadecuadas políticas de producción y altos costos por horas extras. A partir de estas derivaciones se propone diseñar un modelo de gestión de operaciones sobre la base de la programación lineal con la finalidad de determinar el punto óptimo de costo beneficio.

Palabras clave: producción, gestión de operaciones, programación lineal, productos naturales, costos de producción.

\section{Introducción.}

La era de la información, comunicación y redes sociales, ha dado paso a una nueva generación social caracterizada por la concientización ambiental y animal, trayendo consigo varios cambios al mundo actual, que no solo se han visto reflejados en ámbitos sociales, sino políticos, económicos y administrativos; esta conciencia ambiental se percibe llena de oportunidades para un sin número de emprendimientos y demás cambios que las empresas deben sobrellevar para adaptarse al cambiante y exigente mundo actual. Este es el caso del aumento de personas que han optado por un cambio en su régimen alimenticio optando por alimentos que no sean de origen animal. 
Varios estudios indican que el vegetarianismo ha aumentado considerablemente en la última década principalmente en países como Estados Unidos, Canadá, Reino Unido, y otros países Europeos; Aunque en menor medida los países latinoamericanos también son parte de este cambio de pensamiento y específicamente Ecuador no se ha quedado atrás de esta tendencia, en la actualidad es normal encontrar restaurantes con opciones vegetarianas además de establecimientos dedicados completamente a este segmento de mercado, y como es normal el aumento de empresas productoras de estos alimentos.

El objetivo del presente estudio es el desarrollo de un modelo matemático de programación lineal que considere todos los aspectos relevantes a la gestión de operaciones en una empresa productora de productos alimenticios vegetarianos de origen natural. Tras el diseño del modelo matemático el estudio busca resolver un problema muy común en este tipo de empresas que es la reducción de costos de producción con miras a aumentar la rentabilidad del negocio. Para ello se realizó el análisis de cada etapa de un plan de gestión de operaciones, posteriormente se definen las variables que forman parte del modelo de programación lineal para finalmente aplicarla a una unidad de análisis que cumple con las características deseadas. La reducción de los costos de producción, implica un análisis numérico que conlleva a la optimización de los procesos productivos. Según la teoría del padre de la programación lineal George Dantzig cuya hipótesis planteada en 1947 y utilizada en ámbitos administrativos por L.V. Kantoróvich en 1975, la programación lineal es un conjunto de técnicas para la resolución de problemas en decisiones sobre asuntos que intervienen en un gran número de variables, las cuales serán desarrolladas a partir de un plan de gestión de operaciones, con el objetivo ya mencionado de disminución de los costos de producción en empresas que tengan las características mencionadas anteriormente, a continuación, se expone el estado del arte de todos los conceptos necesarios para la elaboración del modelo.

\section{El papel de la programación lineal en la gestión de operaciones.}

La estrecha relación que la programación lineal comparte con el plan de gestión de operaciones, es lo que permitirá establecer una correcta organización en los procesos productivos al aplicar el modelo planteado; existen un sin número de publicaciones relacionadas a la temática que anteceden al estudio y dan valor y realce al mismo. Al respecto, se resaltan las siguientes afirmaciones: las organizaciones excelentes se gestionan mediante procesos estructurados y alineados estratégicamente a partir de decisiones basadas en datos y hechos para obtener resultados equilibrados y sostenidos (Bouza, 2010). Un resultado deseado se alcanza más eficientemente cuando las actividades y los recursos relacionados se gestionan como un proceso (Pesantez, 2016). La gestión por procesos le permite a la organización generar productos o servicios de alta calidad con el fin es satisfacer las necesidades y expectativas de las partes interesadas (Vidal, 2015). La programación lineal se 
la entiende como una técnica que trata la resolución de problemas optimizando los objetivos basados en las variables del problema (Munier, 2011).

\section{Plan de gestión de operaciones:}

El plan de gestión de operaciones que pretende mejorar los procesos productivos tiene además un carácter administrativo, este término académicamente descrito como investigación de operaciones, es la respuesta a la creciente demanda de sistemas de organización tras la revolución industrial. A decir de Hillier y Lieberman (2010): “el objetivo de esta disciplina implica investigar sobre las operaciones. En consecuencia, esta disciplina se aplica a la problemática relacionada con la conducción y la coordinación de actividades en una organización" (p.1). Numerosas investigaciones describen la importancia e impacto de la investigación de operaciones, entre los cuales se aprecian: Mora (2010) la investigación de operaciones tiene un enfoque de optimización y por ello, normalmente se la asocia como una herramienta matemática que en base a los cálculos proporcionados por la minería de datos entregará un valor cuantitativo sobre el estado de las actividades y a partir de ahí permitirá un proceso de mejoramiento. Ramos, Sánchez, Ferrer y Wogrin (2013) expresaron que: "la investigación operativa se puede definir como la aplicación del método científico en la mejora de la efectividad en las operaciones, decisiones y gestión, o como la ciencia de aplicar los recursos disponibles para conseguir la satisfacción optima de un objetivo" (p.3). Díaz (2016) desde una perspectiva científica la gestión es un método que contempla la planificación y ejecución para incrementar la productividad del trabajo.

A decir de Chase, Jacobs, y Aquilano (2010) la gestión o administración de operaciones tiene como objetivo el mejoramiento de un proceso productivo específico desde sus orígenes en el suministro hasta su finalización en el transporte de mercadería. La administración de operaciones es el diseño, operación y mejora continua de sistemas que crean y entregan productos o servicios en una empresa. El diseño de un modelo matemático es el fundamento de la investigación de operaciones, el cual está conformado por factores identificados como problemas al interior de la empresa, el resultado es la solución a dichos inconvenientes y el mejoramiento de los procesos relativos a los mismos (Taha, 2012). La identificación del problema es lo más importante de la gestión de operaciones, más se deberá considerar factores como el comportamiento organizacional en caso de una posible implementación. Es así como un plan de gestión de operaciones se lo esquematiza de forma general como una cadena productiva formada de todos los elementos necesarios para transformar la materia prima a un producto terminado. 
Figura 1. Elementos de un plan de gestión de operaciones.

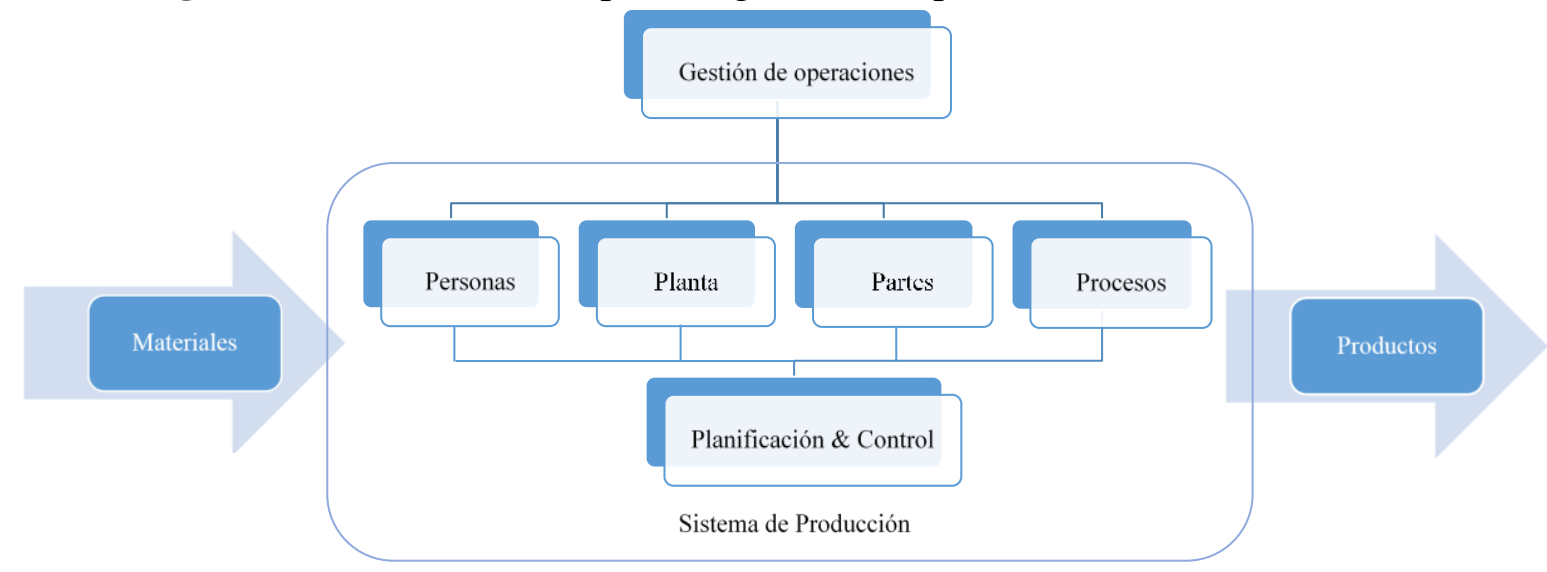

Fuente: Chase, Jacobs, y Aquilano (2010)

Un plan de gestión de operaciones debe ser sistematizado en las diferentes áreas que lo constituyen, determinar un valor numérico para cada una después de identificado los problemas permitirá el diseño de un modelo matemático que pueda ser utilizado para la optimización de los procesos presentes en la cadena productiva. Al respecto, Eppen (2010) afirma que las ecuaciones o expresiones matemáticas están compuestas por términos que son identificables como actividades puntuales del proceso productivo que se requieren optimizar, las mismas que dentro de la expresión matemática forman parte de la función objetivo.

\section{Modelo matemático de programación lineal:}

Los problemas de una empresa son fácilmente identificables tanto por los involucrados en el proceso como por aquellos que pudieran observarlos desde fuera de él, utilizando herramientas de observación directa o por levantamiento de información primaria (encuestas, entrevistas, etc.); Al contrario, plantear matemáticamente un modelo presenta mayor dificultad ya que es necesario encontrar las variables del modelo, las restricciones o limitaciones del mismo y la función objetivo (Taha, 2012). Las variables que se deben identificar para el modelo, son las que señalan la decisión que se debe tomar. En este sentido, Perera, Martín y Martín (2008) señalan que estas variables son "las incógnitas del problema, es decir, las cantidades a fabricar de cada producto para maximizar el beneficio" (p.7). Este concepto trasciende si el objetivo es minimizar costos o encontrar una secuencia de procesos óptima considerando a las variables como las incógnitas del problema independientemente de la naturaleza del proceso o el problema planteado.

En este contexto, resulta conveniente analizar la logística de los procesos productivos, en tal virtud, Mora (2010) afirma que: "La logística es un conjunto de actividades que son repetidas muchas veces a lo largo de la cadena de abastecimiento, desde que las materias primas son convertidas en productos terminados y se agrega valor para los consumidores" (p.35). La creación del modelo matemático no es sencilla, es de suma importancia el entendimiento total de las variables que forman parte de este. A este respecto Eppen (2010) afirma que: 
"Como sabemos, un modelo no es el mundo real, sino una representación abstracta de la realidad. Lo importante para quien formula el modelo es saber cuándo una versión idealizada puede proporcionar una representación adecuada del mundo no lineal" (p.329). El éxito que un modelo tenga dependerá de su correcta implementación y seguimiento.

La programación lineal no debe ser entendida como un término computacional sino más bien como un sinónimo de planificación, adicionalmente el término "lineal", implica que todos los factores matemáticos que conforman la ecuación son funciones lineales, es decir que no tienen valores cuadráticos o de un orden superior que pudieran describir gráficos circulares o parabólicos (Hillier, 2010). Los componentes de un modelo de programación lineal son las variables de decisión las cuales se entiende como las actividades que forman parte del proceso productivo, la función objetivo, que se entiende como el valor que se desea obtener de la suma algebraica de las variables de decisión es decir el aumento en las ganancias o disminución de costos tras realizadas las actividades y las restricciones son aquellas circunstancias que limitan a la función objetivo pudiendo ser de diferente naturaleza, tiempos de máquina, uso de espacios, costos operativos o de mano obra etc. En conjunto las ecuaciones descritas forman el modelo de programación lineal. Para el planteamiento del problema de gestión, primeramente, se debe contar con recursos limitados como equipamiento, trabajadores, dinero, etc. En segundo lugar, el objetivo debe ser explícito, como la intención de reducir costos de producción, adicionalmente las ecuaciones deben ser lineales, los procesos productivos deben ser homogéneos es decir que no existan cambios entre un producto y el siguiente. Finalmente se consideran factores divisibles es decir que la unidad de trabajo tanto para productos como para recursos sea divisible (Chase, 2010). Estas limitaciones serán de gran utilidad al momento de definir las variables y construir el modelo de programación lineal sabiendo de antemano que el proceso alcanzara su punto óptimo posible.

Figura 2. Ejemplo de un modelo matemático de programación lineal y región factible del modelo.

$P=A X_{1}+B X_{2}$

Sujeto a:

$X_{1} \leq 4$

$2 X_{2} \leq 12$

$3 X_{1}+2 X_{2} \leq 18$

$X_{1} \geq 0 \quad y \quad X_{2} \geq 0$

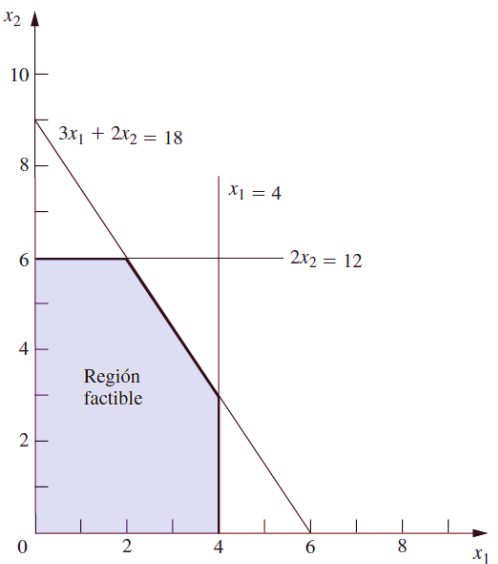


En el ejemplo antes citado la función objetivo $\mathrm{P}$ tiene como finalidad maximizar el resultado que las variables X1 y X2 pueden tener para la empresa, normalmente ligado a la unidad monetaria, los coeficientes que acompañan a las variables, A y B son el redito económico de realizar una sola vez cada actividad y por separado; finalmente las restricciones estipulan limitaciones de fabricación, realización, elementos necesarios que tienen las actividades, entre otros. Estas ecuaciones vienen dadas del análisis y levantamiento de datos de los procesos productivos que conforman un plan de gestión de operaciones. La región factible es aquella representación gráfica de las posibles soluciones a la función objetivo del modelo matemático, se expresa como la intersección de las rectas descritas por las ecuaciones de las restricciones, el resultado para este caso de maximizar la ganancia es el punto más alto que otorgue el mayor redito tras la culminación de las actividades.

El resultado óptimo de la función objetivo se realizará utilizando el método simplex, desarrollado en el año de 1947 por George Dantzig, su uso permite la resolución de problemas complejos por medio de un proceso basado en interacciones, por lo cual, se puede implementar fácilmente en ordenadores (Hillier, 2010). La utilización de este método permite aumentar una cantidad considerable de variables de decisión las mismas que se entienden como valores específicos de un recurso como dinero, tiempo, número de productos, etc. Es importante establecer que el método simplex impone una condición a las ecuaciones de restricciones, y de objetivo al dar por sentado su no negatividad, matemáticamente se entiende esta situación al especificar una condición de desigualdad de las variables que pudiesen dar un valor inferior a cero a la ecuación que las contenga, además se da por entendido que las ecuaciones tienen al menos una solución (Taha, 2012).

Una vez entendido los componentes de un modelo de programación lineal y su función específica dentro del análisis de gestión por operaciones es necesario recalcar como se realiza la construcción del mismo. Las variables se determinan en función de las actividades que son de mayor interés, por lo que, es muy importante su correcta interpretación previo a la inserción de las mismas en el modelo. "Los problemas reales requieren la recolección de los datos relevantes, a menudo la recolección de datos exactos es difícil. Por lo tanto, es común que el valor asignado a un parámetro sea, por necesidad, solo una estimación” (Hillier, 2010, p.10). Estas estimaciones acumularan un error dependiendo del grado de exactitud con el cual se realizó la minería de datos, se recomienda además realizar un análisis de sensibilidad que indique el nivel de certeza que tendrá el modelo.

\section{Análisis de costos de producción:}

En un sentido general se entiende al costo como la cantidad de dinero invertido para la fabricación de un producto, generación de un bien o adquisición de un servicio. Al respecto, Jiménez (2010) señala que: "serán costos los desembolsos causados en el proceso de fabricación o por la prestación de un servicio: sueldos y salarios del personal de la planta de 
producción, materias primas, servicios públicos relacionados con el proceso productivo, etc." (p. 11). En otras palabras, el análisis de costos de producción conlleva todo gasto inherente a la fabricación o creación de un bien, y su estudio permite el cálculo acertado de un precio de venta para el producto final.

Como herramienta para la toma de decisiones e identificación de las variables de un modelo matemático, el estudio de los costos de producción se realiza en base a la contabilidad de costos, la misma que proporciona información para la contabilidad administrativa y para la contabilidad financiera (Horngren y Rajan, 2012). La contabilidad de costos mide, analiza y reporta información financiera y no financiera relacionada con los costos de adquisición o uso de los recursos dentro de una organización. Este enfoque permite utilizar los datos adquiridos de costos como instrumentos de optimización identificando cuáles son las áreas de mayor interés. Para identificar correctamente el costo real de producción, es necesario la elección de un método de costeo, el mismo que se desarrolla en un curso de acción según la clasificación de sus componentes (Rojas, 2014). En este sentido, es importante definir la naturaleza de los costos que se consideran para el modelo.

Los costos generales de producción son aquellos que conllevan la fabricación de un producto, normalmente se consideran la materia prima, costos de mano de obra y de transporte, mas es de suma importancia definir otros costos que también incrementan el precio final del producto o que pudieran afectar el cálculo final. Estos costos adicionales son conocidos como generales o indirectos. A decir de Rojas (2014) los costos generales intervienen en el proceso de transformación de la materia prima hasta la obtención del producto final. De forma general se considera costos operativos, servicios públicos, mantenimiento y políticas de adquisición de maquinaria.

Los costos de mano de obra son aquellos que involucran el pago de sueldos generales, horas extras, pagos de seguros sociales, vacaciones, bonos, utilidades y sueldos extraordinarios de todos los encargados de la transformación de la materia prima en el producto final. Se incluye operadores de maquinaria o personal de planta ya que a decir de Arredondo (2015), no importa que tan automatizada este una planta se considera como costos directos de mano de obra el pago al personal que intervienen en el proceso productivo sin los cuales sería imposible la producción del bien. Es importante considerar para el plan de gestión de operaciones la reducción o la posible eliminación de tiempos no productivos, trabajo indirecto, horas extras.

La materia prima es todo elemento que forma parte integral de un producto, como su nombre lo indica este material se debe poder identificar fácilmente tanto en naturaleza como en costo. Existen dos tipos de materia prima, la utilizada normalmente y en mayor medida conocida como material directo, el segundo tipo de material es el indirecto que no necesariamente será cuantificado con exactitud ya que la cantidad utilizada es muy pequeña o presenta una dificultad considerable para ser medida exactamente. En este contexto, Rojas (2014) afirma 
que "si se observa con detenimiento, para considerar la materia prima como material directo, se deben reunir dos condiciones que son: un importe considerable y saber cuánto de este material hay en cada unidad del producto terminado. Lo anterior obedece al tratamiento que se le da a cada uno de los materiales, ya que el material directo e indirecto tiene tratamiento distinto" (p. 2). Dependiendo del volumen manejado de materia prima se puede agregar el costo de manejo de inventario a este rubro.

Los costos de distribución son parte de los denominados, costos de operación, se consideran valores de comercialización del producto al transporte de material, al personal y a los costos de otras actividades de diversa naturaleza dependiendo del tipo de empresa en la cual se desee hacer el análisis. Las actividades que suman a los costos de producción están dentro de la denominada logística de salida, que incluyen órdenes de compra, embarque de productos o servicios a locales de distribución o clientes finales, ventas por catálogo, por internet etc. (Horngren, 2012). Este análisis es de suma importancia ya que las actividades que lo conforman suelen ser descartadas y un enfoque adecuado permitirá sumar valor comercial al producto, sin afectar desmedidamente el costo final del mismo.

\section{Metodología.}

La presente investigación se desarrolló bajo un diseño no experimental, puesto que no se realizó una manipulación deliberada de las variables de estudio, en tal razón, se observó la determinación de los costos de producción en la unidad de análisis en su contexto natural, para posteriormente analizarlo. En cuanto al enfoque, la investigación se desarrolló bajo el uso de métodos mixtos predominando el análisis cuantitativo sobre el análisis cualitativo, en consecuencia, en una primera fase se describió el fenómeno de estudio, así como las propiedades y características de la gestión de operaciones, la programación lineal y costos de producción y se recolectó información a través de encuestas (dirigidas a los proveedores) y entrevista (al jefe de producción) en la empresa GranOrt; en una segunda fase sobre los resultados construidos del diagnóstico, se interpretó la información obtenida y se elaboró el modelo de programación lineal.

\section{Resultados:}

La empresa GraOrt fue considerada como unidad de análisis ya que se enmarca con las características deseadas para el estudio, se obtuvo la siguiente información como resultado:

- GranOrt es una empresa privada de producción alimentaria, dedicada a la fabricación de alimentos vegetarianos.

- Los productos que ofrece son carne vegetal, granola, barras energéticas y queso de soya, de los cuales la carne vegetal y las barras energéticas son los productos de mayor comercialización.

- La empresa presenta durante el año 2018 un bajo índice de liquidez. 
- Incremento de la cartera de clientes y problemas en el cumplimiento de los pedidos.

- Elevados costos de producción consecuencia de un manejo de inventario inadecuado.

- Deficiente proceso de determinación de los precios de venta.

- Altos costos de mano de obra a consecuencia del incremento de horas extras.

- Falta de tecnificación y políticas de adquisición de maquinaria automatizada.

- El tiempo de respuesta a los clientes por parte de la empresa es aceptable.

- La rotación de los productos en la empresa es media.

- La empresa maneja óptimos procesos de almacenaje, cadena de frio y empaquetado.

- Los proveedores consideran que los precios de comercialización son aceptables.

A partir de los resultados expuestos se presenta el modelo de gestión de operaciones para la empresa GranOrt.

Figura 3. Esquema de los elementos para la propuesta desarrollada.

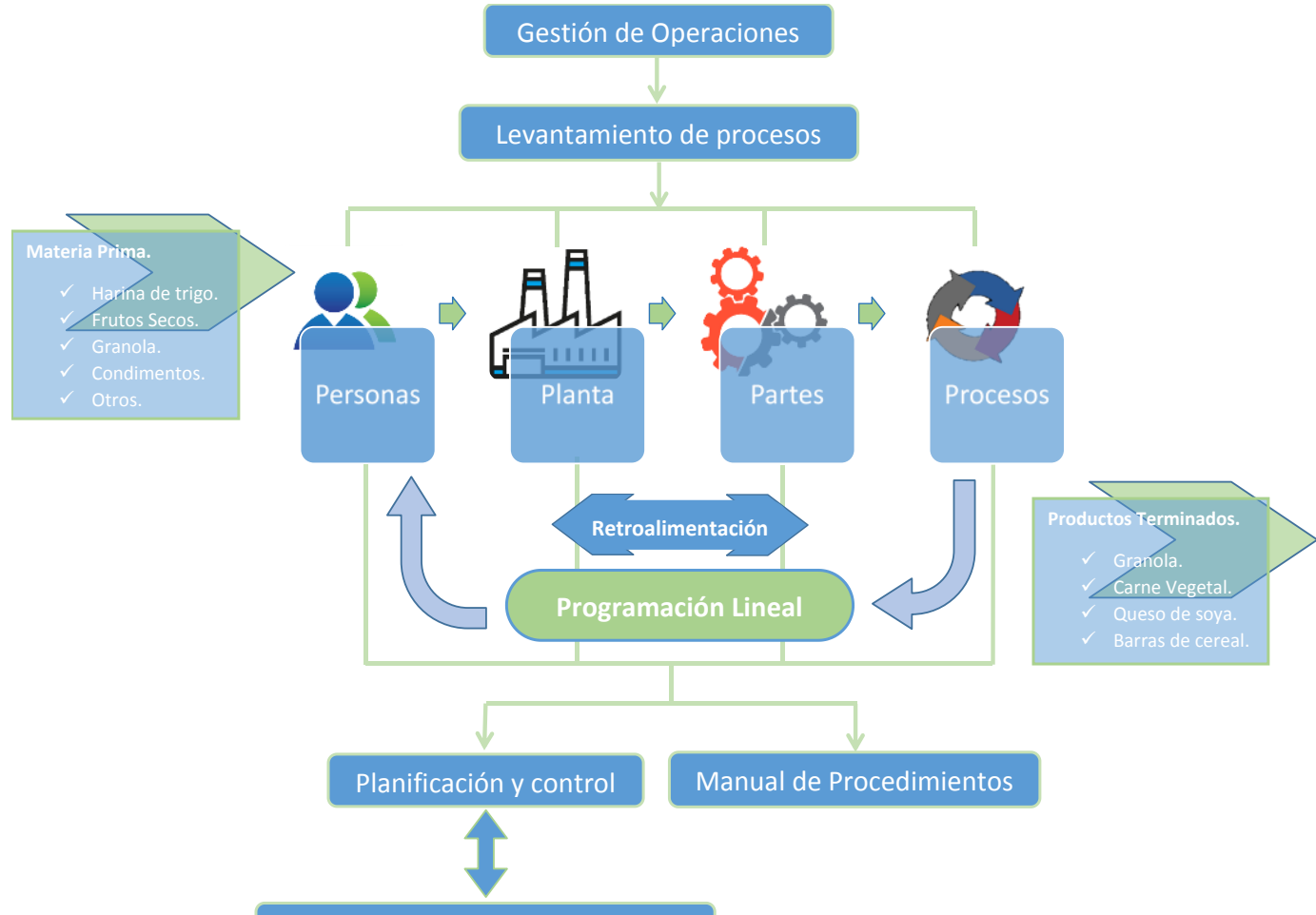

Reducción de costos de producción

Identificado el proceso mediante el cual se puede implementar el plan de gestión de operaciones, se seguirá el camino planteado para determinar estrategias para la reducción en los costos de producción. El primer paso es el levantamiento de procesos productivos los mismos que se encuentran desarrollados en su totalidad en las áreas de soporte, los 
departamentos administrativos que tienen injerencia en la producción, desde el punto de vista de ventas, solicitud de producción y sobre todo cálculo de costos de producción pudieran considerarse en un plan organizacional global o de operaciones administrativas posteriormente. El levantamiento de procesos dio como primer resultado un organigrama estructural que permite observar el flujo de la información y como se realizan las solicitudes de producción. Tanto el organigrama como el proceso para realizar una solicitud de producción, mostrados a continuación son esquemas elaborados tras el levantamiento de información, ya que la empresa carece de los mismos.

Figura 4. Propuesta de organigrama estructural para la empresa GranOrt.

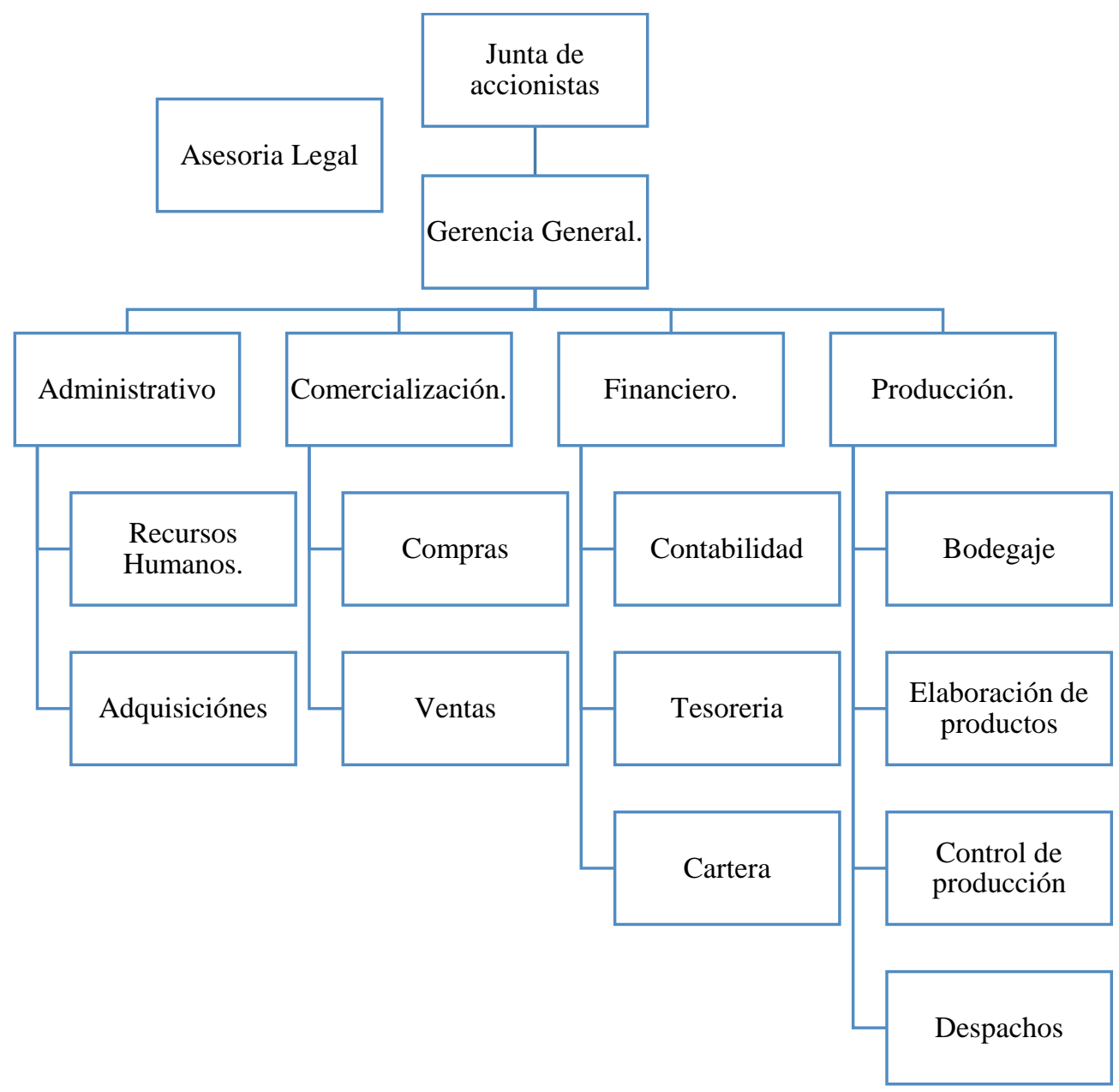

El proceso normal de producción inicia con la solicitud del producto, sea por medio de un pedido directo de uno de los distribuidores, como resultado de una visita de los representantes de ventas o un pedido calendarizado de un contrato de producción, se identifica tipo de producto y cantidad requerida para posteriormente realizar la adquisición de materia prima 
por parte del jefe de producción a uno de los proveedores designados y en la cantidad requerida para solventar el pedido especifico, a continuación se realizan los procesos propios de producción dependiendo del pedido realizado y una vez terminado el proceso se realiza una inspección del producto, un conteo de las unidades requeridas y la verificación de las características del producto versus el pedido, para proceder al empaquetado e identificación mediante, etiquetas, lote, fecha de caducidad y precio de venta, finalmente se realiza la entrega de los productos al distribuidor, conjuntamente con una guía de remisión para verificar lo entregado y dependiendo del tipo y principalmente del volumen del pedido se puede realizar la cobranza el momento de realizar la entrega del producto o después de la misma según la negociación.

Figura 5. Proceso de producción general en la empresa GranOrt.

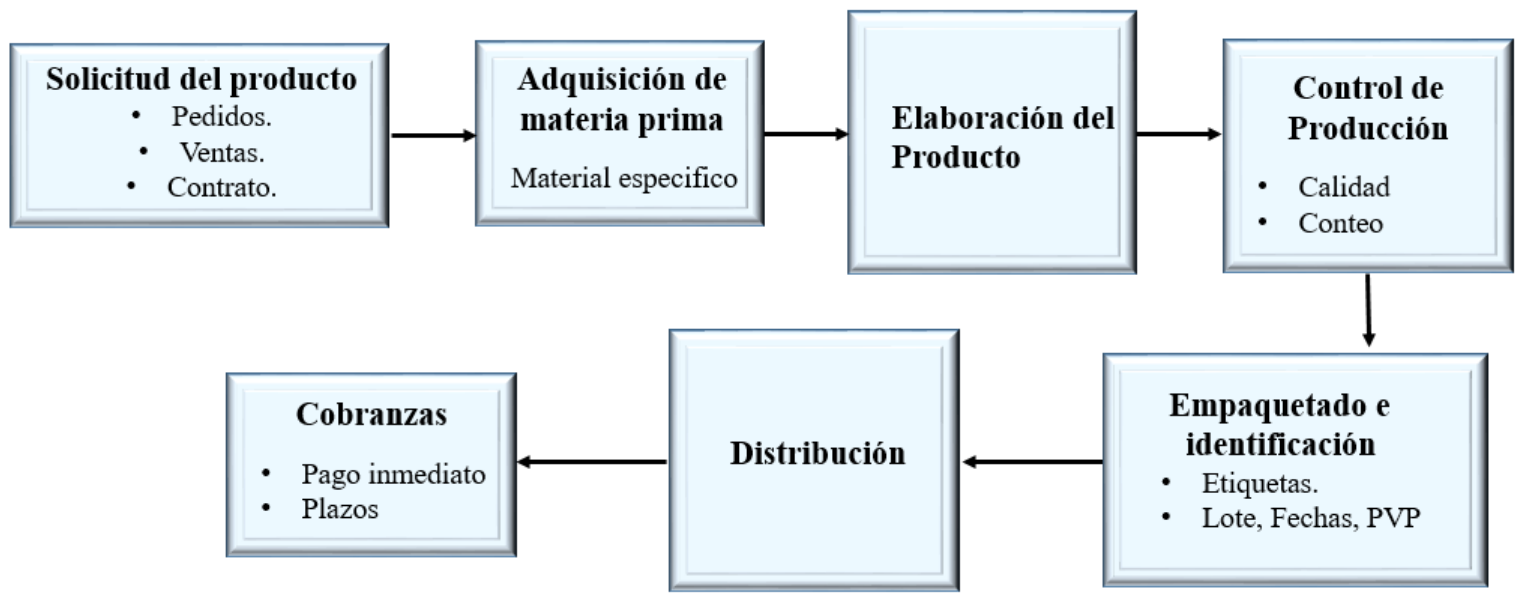

Terminado el levantamiento de información se procede a realizar la identificación de los coeficientes del modelo de programación lineal que se desea implementar. Estos valores serán determinados a partir de las respuestas a la entrevista realizada al jefe de producción conjuntamente con los criterios que especifican los elementos principales del plan de gestión de operaciones, es decir: personas, planta, partes y procesos.

\section{Costos relacionados al personal:}

Como se especificó anteriormente los costos relativos al personal se deben considerar como la suma de todos los pagos realizados a las personas involucradas en el proceso de producción, incluyendo sueldos, bonificaciones, remuneraciones especiales, etc. En base a los estatutos vigentes del ministerio del trabajo al año 2019 de la república del Ecuador en lo concerniente a sueldos y salarios y roles de pago de la empresa se consideran los siguientes valores para los dos involucrados en la producción, cuya remuneración es la base o sueldo mínimo estipulado por la ley de \$394,00 USD para el caso del operador de maquinaria y del jefe de producción que es de \$450,00 USD. 
Tabla 1. Costo total anual del personal de producción.

\begin{tabular}{|c|l|c|c|c|}
\hline No. & \multicolumn{1}{|c|}{ Descripción del costo } & $\begin{array}{c}\text { Costo } \\
\text { unitario }\end{array}$ & $\begin{array}{c}\text { Cantidad } \\
\text { Anual }\end{array}$ & Total \\
\hline $\mathbf{1}$ & Remuneración mensual operador. & $\$ 394,00$ & 12 meses & $\$ 4728,00$ \\
\hline $\mathbf{2}$ & Décimo tercera remuneración operador. & $\$ 394,00$ & 1 Ocasión & $\$ 394,00$ \\
\hline $\mathbf{3}$ & Décimo cuarta remuneración operador. & $\$ 394,00$ & 1 Ocasión & $\$ 394,00$ \\
\hline $\mathbf{4}$ & Fondos de reserva operador. & $\$ 32,83$ & 12 Meses & $\$ 394,00$ \\
\hline $\mathbf{5}$ & Porcentaje de afiliación operador. & $\$ 43,93$ & 12 Meses & $\$ 527,17$ \\
\hline $\mathbf{6}$ & Vacaciones operador. & $\$ 197,00$ & 1 Ocasión & $\$ 197,00$ \\
\hline $\mathbf{7}$ & Remuneración mensual jefe de producción. & $\$ 450,00$ & 12 meses & $\$ 5400,00$ \\
\hline $\mathbf{8}$ & Décimo tercera remuneración jefe. & $\$ 450,00$ & 1 Ocasión & $\$ 450,00$ \\
\hline $\mathbf{9}$ & Décimo cuarta remuneración jefe. & $\$ 394,00$ & 1 Ocasión & $\$ 394,00$ \\
\hline $\mathbf{1 0}$ & Fondos de reserva jefe de producción. & $\$ 37,50$ & 12 Meses & $\$ 450,00$ \\
\hline $\mathbf{1 1}$ & Porcentaje de afiliación jefe de producción. & $\$ 50,18$ & 12 Meses & $\$ 602,10$ \\
\hline $\mathbf{1 2}$ & Vacaciones jefe de producción. & $\$ 225,00$ & 1 Ocasión & $\$ 225,00$ \\
\hline & & \multicolumn{2}{|c|}{ Total } & $\$ 14.155,27$ \\
\hline
\end{tabular}

\section{Costos relacionados a maquinaria e instrumentos.}

Los costos que influyen en el costo final de producción a más de la transformación de la materia prima son los costos por financiamiento con entidades bancarias a largo plazo y la depreciación de las maquinas, equipos, herramientas (ollas de tamaños industrial, centrifuga para la separación del gluten, cocina industrial, empacadora al vacío, horno industrial, congelador vertical, utensilios de cocina, entre otros).

Tabla 2. Costo total anual por depreciación de maquinaria.

\begin{tabular}{|c|l|c|}
\hline No. & \multicolumn{1}{|c|}{ Calculo lineal para la depreciación } & Valor \\
\hline $\mathbf{1}$ & Costo de adquisición. & $\$ 20.000,00$ \\
\hline $\mathbf{2}$ & Costo de venta tras depreciación. & $\$ 7.000,00$ \\
\hline $\mathbf{3}$ & Costo de depreciación. & $\$ 13.000,00$ \\
\hline $\mathbf{4}$ & Tiempo de vida (años) & 5 \\
\hline & Costo total de depreciación anual. & $\$ 2.600,00$ \\
\hline
\end{tabular}

El valor a cancelar mensualmente, por los préstamos relativos a la adquisición de la maquinaria se lo considerará como un único valor mensual debido a la amortización elegida tras la adjudicación del mismo. La suma de estos parámetros dará como resultado el costo total anual en lo que respecta a maquinaria, instrumentos e infraestructura. 
Tabla 3. Costo total anual por planta (maquinaria, instrumentos y espacio físico).

\begin{tabular}{|c|l|c|}
\hline No. & \multicolumn{1}{|c|}{ Cálculo de costos de planta } & Valor \\
\hline $\mathbf{1}$ & Costo total de depreciación anual. & $\$ 2.600,00$ \\
\hline $\mathbf{2}$ & Costo anual del interés bancario. & $\$ 1,100.00$ \\
\hline & Total. & $\$ 3.700,00$ \\
\hline
\end{tabular}

Al igual que los costos relativos al personal, los costos de planta se deberán considerar para un cálculo correcto del precio de venta al público, más en relación al modelo de programación lineal las ecuaciones de restricciones concernientes a este inciso serán diseñadas en base al tiempo maquina necesario para la elaboración de los productos.

\section{Costos relacionados a procedimientos.}

Los costos relativos a procedimientos para la preparación de los productos toman en cuenta los gastos operativos anuales reflejados en el uso de servicios básicos, siendo los únicos insumos adicionales a la materia prima y mano de obra. Se centrará el análisis en el costo de energía eléctrica, compra de gas, telefonía y agua potable mensual, calculados para un año.

Tabla 4. Costos operativos anuales.

\begin{tabular}{|c|l|c|}
\hline No. & Cálculo de costos operativos anuales & Valor \\
\hline $\mathbf{1}$ & Costos anuales por agua potable. & $\$ 600,00$ \\
\hline $\mathbf{2}$ & Costos anuales por energía electica. & $\$ 2.400,00$ \\
\hline $\mathbf{3}$ & Costos anuales por compra de gas. & $\$ 100,00$ \\
\hline $\mathbf{4}$ & Costos anuales por comunicaciones. & $\$ 200,00$ \\
\hline & Costos operativos totales. & $\$ 3.300,00$ \\
\hline
\end{tabular}

El costo de estos servicios básicos podría utilizarse junto con los valores de mano de obra y materia prima para el cálculo correcto del precio de venta al público de los productos, más las restricciones relativas a este parámetro no serán consideradas, ya que las cantidades varían en función de la necesidad y no se pudiera establecer un límite restrictivo a estas.

\section{Costos relacionados a la materia prima.}

Los elementos para la elaboración de los productos ofrecidos por la empresa de estudio son de diferente naturaleza, más existen ciertos elementos que pueden ser utilizados en más de uno; los más importantes son harina de trigo, nueces, pasas, almendras, ojuelos de avena, granos de soya, miel de abeja, harina de maca, sal, pimienta, orégano y stevia. Se considerará un único valor intrínseco en la adquisición de estos proyectados a su producción anual.

Tabla 5. Costo total anual por producto.

\begin{tabular}{|c|l|c|}
\hline No. & Costos inherentes a materia prima & Valor \\
\hline $\mathbf{1}$ & Carne vegetal. & $\$ 6.000,00$ \\
\hline $\mathbf{2}$ & Granola. & $\$ 5.000,00$ \\
\hline $\mathbf{3}$ & Barras de cereal. & $\$ 10.000,00$ \\
\hline $\mathbf{4}$ & Queso de soya. & $\$ 1.500,00$ \\
\hline & Costos totales anuales. & $\$ 22.500,00$ \\
\hline
\end{tabular}


Al no tener un registro detallado de las compras realizadas en materia prima ni históricos de compra a proveedores, el valor mostrado refleja el costo actual de la materia prima que se utiliza para la elaboración de cada unidad de cada producto de acuerdo a la información proporcionada durante la entrevista al jefe de producción y en proyección a un periodo de tiempo de un año. Es decir que se multiplico el costo de una libra de carne vegetal, 400gr de granola, una barra de cereal o una libra de queso de soya por el promedio producido de cada uno mensualmente y después por los doce meses del año. Los datos iniciales como el costo de cada producto son de gran importancia ya que se convertirán en los coeficientes de las variables de decisión de la función objetivo del modelo de programación lineal.

\section{Modelo de programación lineal propuesto.}

La función objetivo debe estar relacionada a los costos de cada producto obtenido durante el levantamiento de información, por consiguiente, minimizar estos costos de producción es la finalidad del estudio. Para ello, es necesario plantear la ecuación de la función objetivo con las restricciones referentes a mano de obra, tiempos de producción y hora máquina necesaria para la elaboración de cada producto. De esta forma el modelo de programación lineal se describiría como se muestra a continuación:

Tabla 6. Variables de decisión y función objetivo del modelo de programación lineal.

\begin{tabular}{|l|c|}
\hline \multicolumn{1}{|c|}{ Variables de decisión } & Función objetivo \\
\hline$X 1=$ Libras de carne vegetal. & \\
$X 2$ & $M I N=Z=1,5 X 1+1,25 X 2+1,25 X 3$ \\
$=$ Unidades de 400 gr de granola. & $+0,25 X 4$ \\
$X 3=$ Libras de queso de soya. & \\
$X 4=$ Barras de cereal. & \\
\hline
\end{tabular}

Cabe recalcar que la unidad de la función objetivo son dólares y tanto los coeficientes de las variables como el resultado, se expresarán en esta unidad. De igual forma es necesario especificar que los procedimientos adicionales que involucran el costo de mano de obra y maquinaria, se visualizarán en las restricciones que complementan el modelo de programación lineal. Estos costos inherentes a personas y planta serán considerados con unidades diferentes no monetarias, debido a que estos no contemplan un valor específico monetario para cada producto. Dicho de otra forma, el valor colocado en las restricciones está en función del tiempo disponible que cada elemento tiene dentro de la planta durante un año calendario sin sobrepasar los costos previamente identificados, los valores de las restricciones serán descritos en la misma unidad: horas, este tiempo refleja la cantidad necesaria que cada producto necesita de cada elemento del plan de gestión de operaciones. 
Tabla 7. Restricciones del modelo de programación lineal.

\begin{tabular}{|c|c|c|c|c|c|c|}
\hline $\begin{array}{c}\text { Gestión de } \\
\text { operaciones }\end{array}$ & Sujeto a: & $\begin{array}{c}\text { Carne } \\
\text { Vegetal } \\
\text { X1 }\end{array}$ & $\begin{array}{c}\text { Granola } \\
\text { X2 }\end{array}$ & $\begin{array}{l}\text { Queso de } \\
\text { soya X3 }\end{array}$ & $\begin{array}{c}\text { Barras } \\
\text { de cereal } \\
\text { X4 }\end{array}$ & Recursos \\
\hline \multirow[t]{2}{*}{ Personas } & $\begin{array}{l}\text { Jefe de } \\
\text { producción }\end{array}$ & 0,124 & 0,125 & 0,440 & 0,010 & 1900 \\
\hline & Operador & 0,092 & 0,085 & 0,305 & 0,005 & 1900 \\
\hline \multirow{6}{*}{ Planta } & $\begin{array}{l}\text { Horno } \\
\text { industrial }\end{array}$ & 0,000 & 0,040 & 0,000 & 0,000 & 1900 \\
\hline & $\begin{array}{l}\text { Centrifuga } \\
\text { de gluten }\end{array}$ & 0,016 & 0,000 & 0,000 & 0,000 & 1900 \\
\hline & $\begin{array}{l}\text { Empacadora } \\
\text { al vacío }\end{array}$ & 0,024 & 0,020 & 0,033 & 0,002 & 1900 \\
\hline & Molino & 0,000 & 0,000 & 0,033 & 0,000 & 1900 \\
\hline & Prensa & 0,000 & 0,000 & 0,066 & 0,000 & 1900 \\
\hline & $\begin{array}{l}\text { Cocina } \\
\text { industrial }\end{array}$ & 0,032 & 0,000 & 0,100 & 0,000 & 1900 \\
\hline
\end{tabular}

Las restricciones se expresan como ecuaciones, las mismas que completan el modelo. A partir de la modelación se realiza el cálculo de la función objetivo por medio de una hoja de cálculo electrónica y la aplicación del método de resolución por iteraciones simplex lineal. Los resultados de la función objetivo mostraran cuantas unidades de cada producto debería fabricarse anualmente para mantener el mínimo de producción requerida, obteniendo el mayor provecho de los recursos.

Tabla 8. Ecuaciones de las restricciones del modelo de programación lineal.

\begin{tabular}{|c|c|}
\hline Restricciones de personas y empaquetado & Restricciones de planta \\
\hline $0,124 X 1+0,125 X 2+0,444 X 3+0,010 X 4$ & $0,033 X 1+0,100 X 3 \leq 1900$ \\
$\leq 1900$ & $0,040 X 2 \leq 1900$ \\
$0,092 X 1+0,085 X 2+0,305 X 3+0,005 X 4$ & $0,016 X 1 \leq 1900$ \\
$\leq 1900$ & $0,033 X 3 \leq 1900$ \\
$0,024 X 1+0,020 X 2+0,033 X 3+0,002 X 4$ & $0,066 X 3 \leq 1900$ \\
$\leq 1900$ \\
\hline \multicolumn{2}{|c|}{ Restricciones de producción } \\
\hline \multicolumn{2}{|c|}{$X 1 \geq 4099,00 ; X 2 \geq 3689,10 ; X 3 \geq 1229,70 ; X 1 \geq 40990,00$}
\end{tabular}

Los valores mostrados en las restricciones de planta, obedecen al hecho de que existe maquinaria especializada utilizada para un solo producto a diferencia de la mano de obra y 
la máquina de empaquetado que se utiliza en todos. Los coeficientes de las restricciones de producción partieron de un valor obtenido durante el levantamiento de datos.

Figura 6. Parámetros de la herramienta solver para la resolución del modelo de programación lineal.

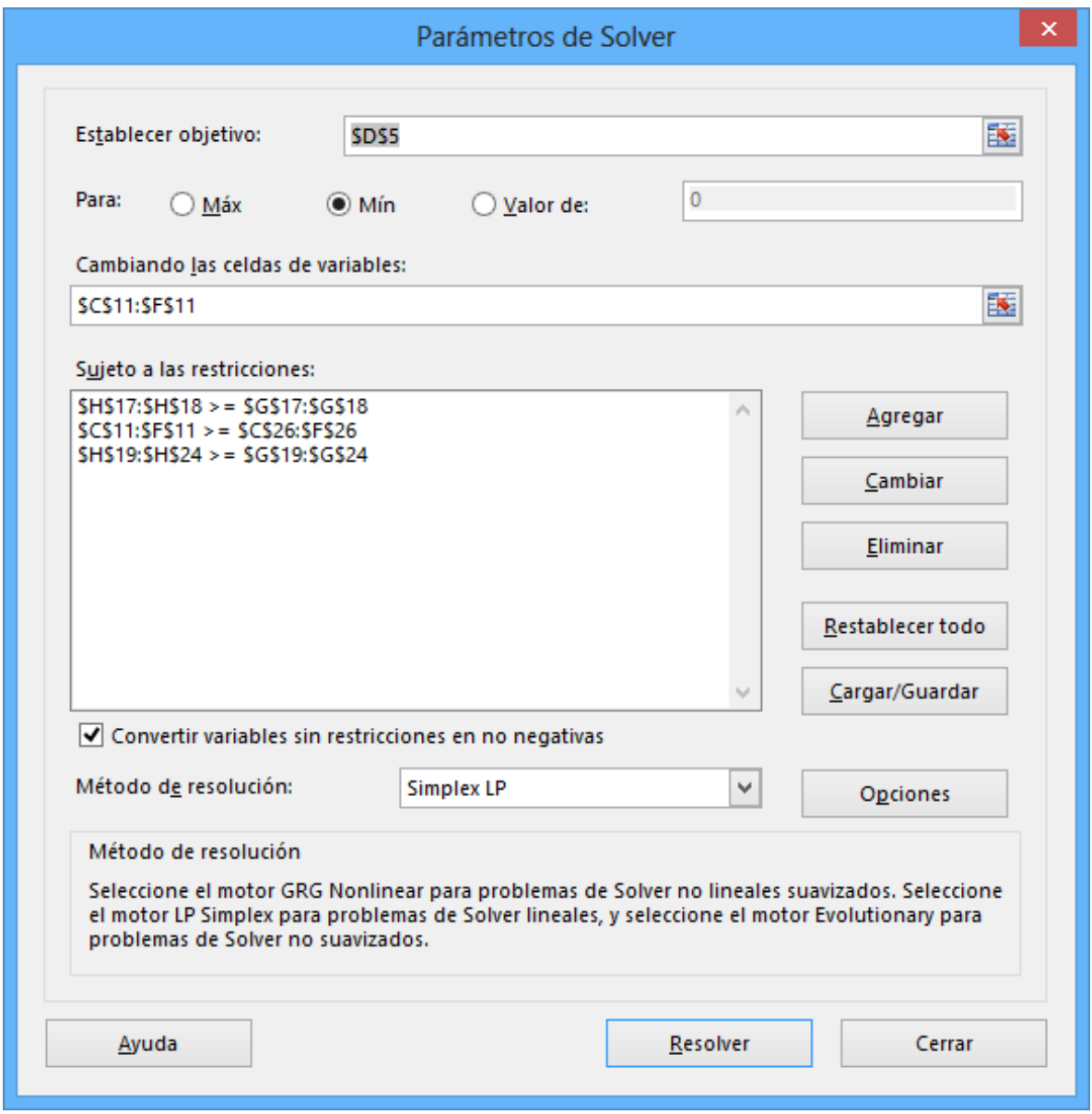

Las restricciones fueron agregadas a la hoja de cálculo por medio de la herramienta solver ${ }^{5}$, como se muestra en la figura anterior, donde se especifica además las celdas para el cálculo de la función objetivo y el valor para las variables de decisión.

\footnotetext{
${ }^{5}$ Es un complemento del programa Microsoft Excel, usado para el cálculo del valor óptimo y análisis de datos.
} 
Figura 7. Resolución del modelo de programación lineal propuesto.

\begin{tabular}{|c|c|c|c|}
\hline \multicolumn{4}{|c|}{ Funcion objetivo } \\
\hline & \multicolumn{2}{|c|}{ Resultado obtenido: } & \\
\hline $\mathbf{Z}$ & \multicolumn{2}{|c|}{22544,5} & $\mathbf{M I N}=\mathbf{Z}=1,5 \mathbf{X 1}+1,25 \mathbf{X} \mathbf{2}+1,25 \mathbf{X} \mathbf{3}+0,25 \mathbf{X} \mathbf{4}$ \\
\hline \multicolumn{4}{|c|}{ Variables de decisión } \\
\hline Carne Vegetal & Granola & Queso de soya & Barras de Cereal \\
\hline $\mathrm{X} 1$ & $\mathrm{X} 2$ & $\mathrm{X} 3$ & $\mathrm{X} 4$ \\
\hline 4099 & 3689,1 & 1229,7 & 40990 \\
\hline \multicolumn{4}{|c|}{ Restricciones } \\
\hline
\end{tabular}

\begin{tabular}{|c|c|c|c|c|c|c|}
\hline \\
\hline \multirow{3}{*}{$\begin{array}{c}\text { Suejeto a: } \\
\text { Jefe de producción }\end{array}$} & & & & & \multirow{3}{*}{$\begin{array}{c}\text { Recursos } \\
\text { Utilizados } \\
1899,89\end{array}$} & \multirow{3}{*}{$\begin{array}{c}\text { Recursos } \\
\text { Disponibles } \\
1900\end{array}$} \\
\hline & & & & & & \\
\hline & 0,124 & 0,125 & 0,440 & 0,010 & & \\
\hline Operador & 0,092 & 0,085 & 0,305 & 0,005 & 1270,69 & 1900 \\
\hline Horno Industrial & 0,000 & 0,040 & 0,000 & 0,000 & 147,56 & 1900 \\
\hline Centrifuga de gluten & 0,016 & 0,000 & 0,000 & 0,000 & 65,58 & 1900 \\
\hline Empacadora al vacio & 0,024 & 0,020 & 0,033 & 0,002 & 294,72 & 1900 \\
\hline Molino & 0,000 & 0,000 & 0,033 & 0,000 & 40,58 & 1900 \\
\hline Prensa & 0,000 & 0,000 & 0,066 & 0,000 & 81,16 & 1900 \\
\hline Cocina industrial & 0,032 & 0,000 & 0,100 & 0,000 & 254,14 & 1900 \\
\hline
\end{tabular}

\begin{tabular}{l|c|c|c|r} 
Minimo & 4099,00 & 3689,10 & 1229,70 & 40990,00
\end{tabular}

Las celdas resaltadas son los valores calculados por el algoritmo de resolución simplex interno en la hoja de cálculo. Se identifican tres áreas de interés: primero los recursos utilizados, que se entienden como la cantidad en horas que se necesita de cada elemento del plan de gestión de operaciones para lograr el mínimo referido por la empresa, segundo, el valor en unidades de cada producto ofrecido por la empresa que se quiere realizar cumpliendo el mínimo solicitado y, tercero, el valor económico mínimo posible para alcanzar el objetivo, es decir, el resultado de la función objetivo. Los resultados evidencian que el modelo se acoplo a los requisitos de la empresa respetando los valores máximos de costos de producción planteados y mostrando exactamente cuál debería ser la producción para minimizar los costos de producción. A partir de los resultados se pueden implementar varias estrategias que reduzcan aún más el costo de producción o incrementen la ganancia una vez implementado el plan de gestión de operaciones. 


\section{Conclusiones:}

Un plan de gestión de operaciones es una herramienta que permite la identificación completa de todos los procesos productivos en una empresa, definirlos de manera correcta posibilita la adecuada interpretación del estado actual de un área productiva y la optimización de aquellas actividades donde se encuentran falencias u oportunidades de mejora.

El modelo de programación lineal apropiadamente interpretado y diseñado especifica valores puntuales que otorgan información valiosa para la toma de decisiones o el planteamiento de políticas de producción, añadido a un plan de gestión de operaciones forman un instrumento de gran valor para un área productiva, cualquiera que sea el origen u objetivo de la empresa.

La herramienta de entrevista utilizada para el levantamiento de datos, permitió el cálculo correcto de los costos de producción, que se convirtieron en los coeficientes de la función objetivo del modelo de programación lineal. Los costos, así como la producción mínima requerida admitida por el jefe de producción fueron esenciales para el cumplimiento del objetivo planteado en la unidad de análisis y de gran ayuda para dar realce a la investigación.

Las encuestas realizadas a los distribuidores ampliaron la visión del estudio, trascendiendo del modelo de programación y dando mayor realce al plan de gestión de operaciones ya que la información recogida permitirá por si sola la aplicación de ciertas políticas y estrategias de producción que beneficiaran a la unidad de análisis y que pudieran replicarse a otras empresas de igual o similar naturaleza.

El resultado del modelo de programación lineal (función objetivo) ratifica que el mínimo de producción solicitado por la unidad de análisis es posible lograrlo sin tener que incurrir en costos adicionales por concepto de horas extras, para ello, se sugiere la implementación de un programa de producción basado en proyecciones, y el aprovechamiento de las ventajas en términos de costos bajo una producción de economías de escala, sustentada en compra a gran escala de materia prima, incremento de la especialización de los gestores, obtención de fuentes de financiamiento a bajos costos e incorporación de nuevas tecnologías.

Plantear un modelo matemático puntual considerando elementos del plan de gestión de operaciones permitió una visión más específica y sostenida sobre valores de los componentes que afectan económicamente a la transformación de la materia prima, además, propicio el cálculo de los valores necesarios para proyectar de manera correcta la producción, permitiendo una mejor administración de toda la cadena productiva. 


\section{Referencias Bibliográficas:}

Arredondo Gonzáles, M. M. (2015). Contabilidad y Análisis de Costos. México D.F.: Grupo Editorial Patria.

Bouza, O. (2010). Desarrollo del ámbito informacional desde la perspectiva de la sistematización de la Vigilancia Científica y Tecnológica (VCT) en organizaciones empresariales. Granada: Editorial de la Universidad de Granada.

Chase, R., Jacobs, F. R., \& Aquilano, N. (2010). Administración de Operaciones producción y cadena de suministros. México: McGraw-Hill.

Díaz, A. (2016). Modelo de gestión comercial estratégico para el centro de llamadas de plasticaucho industrial S.A. Ambato: Pontificia Universidad Católica del Ecuador.

Eppen, G. D., Gould, F. J., Schmidt, C. P., Moore, J. H., \& Weatherford, L. R. (2000). Investigación de Operaciones en la Ciencia Administrativa. México: Prentice-hall.

Gessa Perera, A., Rabadán Martín, I., \& Jurado Martín , J. A. (2008). Aplicación de la programación lineal en la planificación de la producción. Implicaciones del Protocolo de Kioto. II International Conference on Industrial Engineering and Industrial Management, 7-10.

Hillier, F., \& Gerald, L. (2010). Introducción a la Investigación de Operaciones. México: McGraw-Hill.

Horngren, C. T., Datar, S. M., \& Rajan, M. V. (2012). Contabilidad de costos, Un enfoque gerencial. México: Pearson.

Jiménez, W. (2010). Contabilidad de Costos. Bogotá: Fundación para la Educación Superior San Mateo.

Mora García, L. A. (2010). Gestión Logística Integral. Bogotá: Ecoe Ediciones.

Munier, N. (2011). Procedimiento fundamentado en la programación lineal para la selección de alternativas en proyectos de naturaleza compleja y con objetivos múltiples. Valencia: Editorial de la Universidad de Valencia.

Pesántez, C. (2016). Modelo de gestión por procesos basado en la norma ISO 9001:2008 Aplicado a la empresa Compufácil. Cuenca: Editorial de la Universidad Politécnica Salesiana del Ecuador. 
Ramos, A., Sánchez, P., Ferrer, J., \& Wogrin, S. (2013). Modelos matemáticos de optimización. Madrid: Universidad Pontificia Comillas.

Rojas Medina, R. (2014). Contabilidad de Costos. Bogotá: Pontificia Universidad Javeriana.

Taha, H. A. (2012). Investigación de Operaciones. México D.F.: Pearson.

Vidal, E. (2015). Metodología para la elaboración de un modelo de gestión aplicado a fondos complementarios previsionales cerrados de cesantía del Austro Ecuatoriano. Cuenca: Editorial de la Universidad del Azuay.

\section{U Ciencia}




\section{PARA CITAR EL ARTÍCULO INDEXADO.}

Escandón Cueva, R., Erazo Álvarez, J., Narváez Zurita, C., \& Ormaza Andrade, J. (2019). La gestión de operaciones como herramienta generadora de ventajas competitivas en la industria alimentaria vegetariana. Visionario $\quad$ Digital, 3(2.2), 28-49. https://doi.org/10.33262/visionariodigital.v3i2.2.597

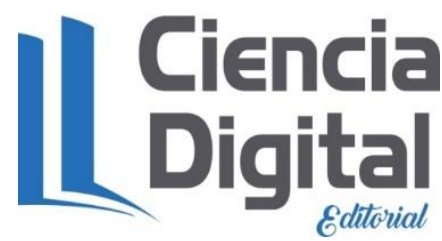

El artículo que se publica es de exclusiva responsabilidad de los autores y no necesariamente reflejan el pensamiento de la Revista Ciencia Digital.

El artículo queda en propiedad de la revista y, por tanto, su publicación parcial y/o total en otro medio tiene que ser autorizado por el director de la Revista Ciencia Digital.
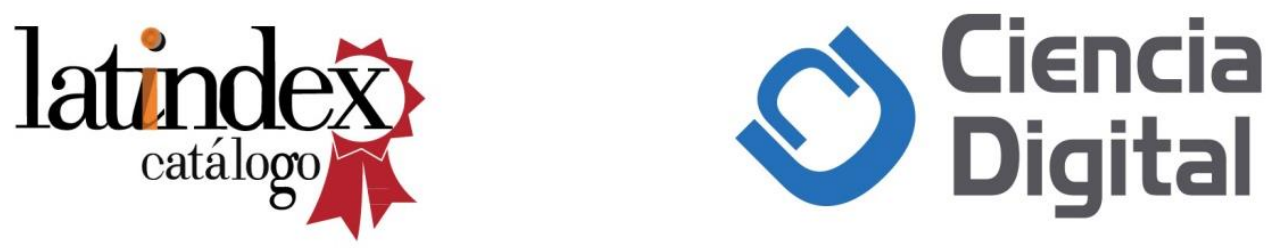